\title{
Correlation of BAW and SAW properties of langasite at elevated temperatures
}

\author{
M. Schulz ${ }^{1}$, E. Mayer ${ }^{2}$, I. Shrena ${ }^{2}$, D. Eisele ${ }^{2}$, M. Schmitt ${ }^{2}$, L. M. Reindl ${ }^{2}$, and H. Fritze ${ }^{1}$ \\ ${ }^{1}$ Institute of Energy Research and Physical Technologies, Clausthal University of Technology, Goslar, Germany \\ ${ }^{2}$ Department of Microsystems Engineering, Albert-Ludwigs-Universität Freiburg, Freiburg, Germany
}

Correspondence to: M. Schulz (michal.schulz@tu-clausthal.de)

Received: 8 October 2015 - Accepted: 27 November 2015 - Published: 17 December 2015

\begin{abstract}
The full set of electromechanical data of langasite $\left(\mathrm{La}_{3} \mathrm{Ga}_{5} \mathrm{SiO}_{14}\right)$ is determined in the temperature range from 20 to $900^{\circ} \mathrm{C}$ using differently oriented bulk acoustic wave resonators. For data evaluation a physical model of vibration is developed and applied. Thereby, special emphasis is taken on mechanical and electrical losses at high temperatures. The resulting data set is used to calculate the properties of surface acoustic waves. Direct comparison with experimental data such as velocity, coupling coefficients and propagation loss measured using surface acoustic wave devices with two different crystal orientations shows good agreement.
\end{abstract}

\section{Introduction}

Langasite (LGS, $\mathrm{La}_{3} \mathrm{Ga}_{5} \mathrm{SiO}_{14}$ ) is a single crystalline piezoelectric material suited for high-temperature applications (Fukuda et al., 1998; Fachberger et al., 2001; Fritze and Tuller, 2001; Fritze, 2011b). It belongs to the point group 32 and exhibits the same crystal structure as quartz, but exceeds its operation temperature limit significantly. LGS does not undergo any phase transformation up to its melting point at $1470{ }^{\circ} \mathrm{C}$ and may be piezoelectrically excited up to at least $1400^{\circ} \mathrm{C}$ provided that stable electrodes are available (Fritze, 2011b). The crystals exhibit mixed electronic and ionic conductivity, which contributes to the loss at high temperatures. The oxygen partial pressure-dependent conductivity impacts the performance of the resonators in a minor way as long as the oxygen partial pressure is kept, e.g., above $10^{-20}$ bar at $600{ }^{\circ} \mathrm{C}$ (Fritze et al., 2006; Fritze, 2006, 2011a).

LGS can be used as a resonant sensor. When operated in the microbalance mode, small mass changes of a layer with affinity to specific gas particles cause a shift of the resonance frequency (Tuller and Fritze, 2002). Anticipated applications of such sensors include fuel cells and gas reformers (Schneider et al., 2005). The stability of LGS in harsh environments such as high temperatures and low oxygen partial pressures makes it suited for wireless sensors based on surface acoustic wave (SAW) devices (Fachberger et al., 2004; Thiele and Pereira da Cunha, 2006; Bardong et al., 2008;
Wall et al., 2015; Behanan et al., 2015). Here, an accurate set of LGS material constants determined for a wide temperature range is strongly required. The availability of such data sets is scarce, since the published materials data are either limited to a fixed temperature or cover only a narrow range around room temperature ${ }^{\circ} \mathrm{C}$ (Bungo et al., 1999; Malocha et al., 2000). Weihnacht et al. (2012) provide the components of elastic compliance tensor in the 25 to $600{ }^{\circ} \mathrm{C}$ temperature range, determined using the pulse-echo ultrasonic technique (Weihnacht et al., 2012).

In this work, the full set of elastic, electric and piezoelectric properties of langasite is determined at elevated temperatures using bulk acoustic wave (BAW) resonators of different orientations. Special attention is drawn to the mechanical and electrical losses, which play an important role for high-temperature applications. Subsequently, the full set of electromechanical data is used to predict the wave velocity of SAW resonators for two selected cuts of LGS. Their Euler angles are $\left(0,138.5,26.6^{\circ}\right)$ and $\left(0,30.1,26.6^{\circ}\right)$. Finally, the calculated values are compared with the corresponding data extracted from the high-temperature measurements of specially designed SAW test structures.

The LGS crystals for BAW resonators are provided by the Institute for Crystal Growth, Berlin Adlershof, Germany. The wafers for SAW measurements are fabricated by FOMOS- 
Materials, Russia, and Mitsubishi Materials Corporation, Japan.

\section{Model for BAW and SAW}

\subsection{High-temperature losses}

A piezoelectric resonator operated at room temperature can be described by a one-dimensional physical model of vibration, where electrical losses are very low and thus negligible. At elevated temperatures, this description is not accurate due to, e.g., the finite electrical conductivity. In this case the elastic, dielectric and piezoelectric coefficients must be extended by imaginary parts which express the mechanical, electrical and piezoelectric losses.

\subsubsection{Mechanical loss}

The mechanical loss at elevated temperatures depends on the resonance frequency $f$ and can be described by the viscosity $\eta$. The loss of the resonator at the angular frequency $\omega$, with $\omega=2 \pi f$, is related to the imaginary part of the elastic stiffness according to (Ikeda, 1990):

$\hat{c}=c+j \omega \eta$.

The elastic compliance $s$ must be extended by a viscous contributions in a similar way. The relation between the elastic stiffness and the viscosity can be expressed using the inverse resonant quality factor, $Q^{-1}$ and the mechanical loss tangent $\tan \delta_{\mathrm{M}}$, by

$\tan \delta_{\mathrm{M}}=\frac{\Im(\hat{s})}{\Re(\hat{c})}=\frac{\omega \eta}{c} \equiv Q^{-1}$.

\subsubsection{Electrical loss}

A perfectly insulating resonator with two parallel electrodes, excited by a harmonic electric field $E=E_{0} e^{j \omega t}$, acts as a capacitor with a dielectric material described by the permittivity $\varepsilon$. In case of materials exhibiting dielectric loss, the dielectric tensor must be regarded as a complex property consisting of real and imaginary parts (Ikeda, 1990)

$\hat{\varepsilon}=\varepsilon-j \frac{\sigma}{\omega}$,

with the loss $\operatorname{tangent} \tan \delta_{E}$ given by

$\tan \delta_{E}=\frac{\Im(\hat{\imath})}{\Re(\hat{e})}$.

The origin of dielectric loss can be attributed to the electrical conductivity $\sigma$ of the material. Calculating the admittance $Y$ for a capacitor with the thickness $a$, the electrode area $A$ and the dielectric coefficient described by the complex property $\hat{\varepsilon}$ results in (Ikeda, 1990)

$Y=\frac{A}{a}(\sigma+j \omega \varepsilon)$.
The equation describes the admittance of an electric circuit with capacitor and resistance connected in parallel.

\subsubsection{Piezoelectric loss}

In analogy to the mechanical and electrical loss, the imaginary part of the piezoelectric coefficient can be introduced. The nature of this "piezoelectric loss" is however not clear. It may be explained by, e.g., jumping of lattice defects or the movement of domain walls in polycrystalline materials (Sherrit and Mukherjee, 1998; Smits, 1976). However, for most materials this term is negligible and assumed to be zero (Ikeda, 1990). The validity of this assumption for langasite is proven in previous studies, where calculations with complex piezoelectric coefficient are used to evaluate the data (Fritze, 2011b). Therefore, in this article the imaginary part of the piezoelectric coefficient is omitted.

\subsection{Piezoelectric equations}

In case of piezoelectric materials, the relation between mechanical and electrical properties is described by the piezoelectric equation

$\left[\begin{array}{cc}\hat{c} & e^{T} \\ e & \hat{\varepsilon}\end{array}\right] \cdot\left[\begin{array}{c}S \\ E\end{array}\right]=\left[\begin{array}{l}T \\ D\end{array}\right]$,

where the complex stiffness $\hat{\boldsymbol{c}}$ and the piezoelectric constant $\boldsymbol{e}$ are 4th and 3rd rank tensors, respectively. The complex dielectric coefficient $\hat{\boldsymbol{\varepsilon}}$, strain $\boldsymbol{S}$ and stress $\boldsymbol{T}$ are 2nd rank tensors. The electric field $\boldsymbol{E}$ and electric displacement $\boldsymbol{D}$ are vectors. In order to differentiate between tensors and tensor components, the former is written in bold.

Due to the tensor symmetry and the crystal symmetry of LGS many tensor components vanish, so that the number of independent coefficients in Eq. (6) is reduced to 10 (Meitzler et al., 1988; Ikeda, 1990):

$\left[\begin{array}{ccccccccc}\hat{c}_{11} & \hat{c}_{12} & \hat{c}_{13} & \hat{c}_{14} & 0 & 0 & e_{11} & 0 & 0 \\ \hat{c}_{12} & \hat{c}_{11} & \hat{c}_{13} & -\hat{c}_{14} & 0 & 0 & -e_{11} & 0 & 0 \\ \hat{c}_{13} & \hat{c}_{13} & \hat{c}_{33} & 0 & 0 & 0 & 0 & 0 & 0 \\ \hat{c}_{14} & -\hat{c}_{14} & 0 & \hat{c}_{44} & 0 & 0 & e_{14} & 0 & 0 \\ 0 & 0 & 0 & 0 & \hat{c}_{44} & \hat{c}_{14} & 0 & -e_{14} & 0 \\ 0 & 0 & 0 & 0 & \hat{c}_{14} & \hat{c}_{66} & 0 & -e_{11} & 0 \\ e_{11} & -e_{11} & 0 & e_{14} & 0 & 0 & \hat{\varepsilon}_{11} & 0 & 0 \\ 0 & 0 & 0 & 0 & -e_{14} & -e_{11} & 0 & \hat{\varepsilon}_{11} & 0 \\ 0 & 0 & 0 & 0 & 0 & 0 & 0 & 0 & \hat{\varepsilon}_{33}\end{array}\right]$,

with $\hat{c}_{66}=\left(\hat{c}_{11}-\hat{c}_{12}\right) / 2$. Here, the $\hat{c}_{i j}, \hat{e}_{i j}$ and $\hat{\varepsilon}_{i j}$ represent the components of stiffness tensor $\boldsymbol{c}$, piezoelectric tensor $\boldsymbol{e}$ and dielectric coefficient tensor $\varepsilon$, respectively.

Equation (6) applies, when the strain $S$ and electric field $\boldsymbol{E}$ are independent variables. This is the case where a resonator is operated in the thickness shear mode of vibration. In case of length-extensional mode of vibration, where the elastic stress $\boldsymbol{T}$ and the electric field $\boldsymbol{E}$ are independent variables, an alternative notation applies

$\left[\begin{array}{cc}\hat{s} & d^{T} \\ d & \hat{\varepsilon}\end{array}\right] \cdot\left[\begin{array}{c}T \\ E\end{array}\right]=\left[\begin{array}{l}S \\ D\end{array}\right]$. 
Here, the complex elastic compliance $\hat{s}$ is used instead of the stiffness $\hat{\boldsymbol{c}}$, and the piezoelectric constant $\boldsymbol{d}$ replaces the coefficient $\boldsymbol{e}$. The relation between piezoelectric tensors $\boldsymbol{e}$ and $\boldsymbol{d}$ and the relation between elastic compliance and stiffness are shown, e.g., in (Meitzler et al., 1988; Ikeda, 1990). The latter is used to calculate the component $s_{12}(T)$ of the elastic stiffness tensor from the coefficient $c_{66}(T)$ as follows:

$s_{12}(T)=s_{11}(T)-\frac{1}{2 c_{66}(T)}-\frac{2\left(s_{14}(T)\right)^{2}}{s_{44}(T)}$.

The determination of all components of the elastic stiffness and compliance tensors requires different crystal cuts. The shear components of those tensors determine two different modes of vibration. The stiffness $\hat{c}_{66}$ describes the thickness shear (TS) vibration of a partially electroded $Y$ cut resonator, and the elastic compliance $\hat{s}_{44}$ describes the face shear (FS) vibration of a rectangular $Y$-cut plate. The remaining four components of the elastic compliance tensor may be determined using differently oriented rods excited in length-extensional (LE) mode of vibration. Here, the effective compliance $s^{\text {eff }}$ is a superposition of several components as function of the angle $\varphi$ between the rod and the $Y$ axis (Ikeda, 1990; Schulz and Fritze, 2008):

$$
\begin{aligned}
s^{\text {eff }}(\varphi) & =s_{11} \cos ^{4} \varphi+s_{33} \sin ^{4} \varphi-2 s_{14} \cos ^{3} \varphi \sin \varphi \\
& +\left(s_{44}+2 s_{13}\right) \cos ^{2} \varphi \sin ^{2} \varphi .
\end{aligned}
$$

Several angles $\varphi$ have to be chosen in such a way that for every $\varphi$ at least one term in Eq. (10) dominates. The effective piezoelectric coefficient $d^{\text {eff }}$ is a superposition of two different coefficients as a function of the $\varphi$ :

$d^{\text {eff }}(\varphi)=-d_{11} \cos ^{2} \varphi+d_{14} \cos \varphi \sin \varphi$.

From Eq. (10) it may be seen that four differently oriented rods with angles $\varphi$ of $-30,0,30$ and $45^{\circ}$ are required to determine the majority of components of the elastic compliance $\hat{s}$. The coefficient $s_{12}$ remains to be determined and is obtained from the thickness shear mode of vibration as shown in Eq. (9). The sum of coefficients $\left(s_{44}+2 s_{14}\right)$ is separated using the face shear mode of vibration described by $s_{44}$. The stiffness tensor $\hat{\boldsymbol{c}}$ is calculated using the relation between stiffness and elastic compliance. Additionally, two rectangular $X$ - and $Z$-cut plates are required to determine the two independent complex components of the dielectric tensor, $\hat{\varepsilon}_{11}$ and $\hat{\varepsilon}_{33}$. The imaginary part of dielectric tensor provides the information about conductivity $\sigma_{1}$ and $\sigma_{3}$ (commonly denoted as $\sigma_{X}$ and $\sigma_{Z}$, respectively) of langasite.

All crystal cuts used in this work are visualized in Fig. 1.

\subsection{Models for different crystal orientations}

\subsubsection{Length-extensional mode}

In case of a rod, where the width $b$, length $l$ and thickness $a$ are chosen to be parallel to the $x_{3}, x_{2}$ and $x_{1}$ axes, respectively, and the applied electric field is parallel to the $x_{1}$ axis,

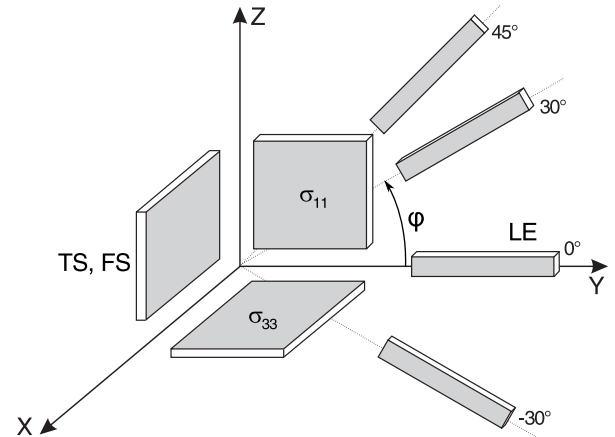

Figure 1. The crystal cuts of langasite used to determine the full set of electromechanical data (Ikeda, 1990). Four differently rotated rods with electrodes perpendicular to $X$ axis vibrate in length extensional mode (LE). The rectangular $Y$-cut plate vibrates in face shear (FS) and thickness shear (TS) modes. The rectangular $X$ - and $Z$-cut plates are used for conductivity measurements.

the piezoelectric relation shown in Eq. (8) is reduced significantly. Due to the boundary conditions chosen here, only the stress and strain in $x_{2}$ direction are relevant. All other components of the tensors $\boldsymbol{T}$ and $\boldsymbol{S}$ vanish. Similarly, only the electric displacement $D_{1}$ and electric field $E_{1}$ are taken into account resulting in the following equation:

$S_{2}=\hat{s}_{22} T_{2}+d_{12} E_{1}$

$D_{1}=d_{12} T_{2}+\hat{\varepsilon}_{11} E_{1}$.

The strength of the coupling between the mechanical and electrical properties of a piezoelectric medium is expressed as coupling factor $\hat{k}$, defined by

$\hat{k}_{12}^{2}=\frac{d_{12}^{2}}{\hat{s}_{22} \hat{\varepsilon}_{11}}$.

With the coupling factor, Eq. (12) can be transformed in

$$
\begin{aligned}
& T_{2}=\frac{1}{\hat{s}_{22}} \frac{\partial u_{2}}{\partial x_{2}}-\frac{d_{12}}{\hat{s}_{22}} E_{1}, \\
& D_{1}=\frac{d_{12}}{\hat{s}_{22}} \frac{\partial u_{2}}{\partial x_{2}}+\hat{\varepsilon}_{11}\left(1-\hat{k}_{12}^{2}\right) E_{1} .
\end{aligned}
$$

Solving Newton's equation of motion with $T_{2}$ described by Eq. (14) and under the assumption of harmonic time dependence $e^{j \omega t}$ of the electric field $E_{1}$, the electric impedance of the resonator is calculated (Schulz and Fritze, 2008)

$$
Y=j \omega \frac{l b}{a} \hat{\varepsilon}\left[1-\hat{k}^{2}+\hat{k}^{2} \frac{2}{l b \sqrt{\rho \hat{s}_{22}}} \tan \left(\frac{l b \sqrt{\rho \hat{s}_{22}}}{2}\right)\right] .
$$

The parameter of the tangent function reflects the phase shift and a wave velocity in the bulk of a resonator:

$\hat{v}=l b \sqrt{\rho \hat{s}_{22}}$. 
The capacity $C_{0}$ of the resonator equals

$C_{0}=\hat{\varepsilon}_{11} \frac{l b}{a}$.

From Eqs. (15), (16) and (17) the admittance $Y$ of a resonator is found to be

$Y=j \omega C_{0}\left[1-\hat{k}^{2}+\hat{k}^{2} \frac{2}{\hat{v}} \tan \left(\frac{\hat{v}}{2}\right)\right]$.

\subsubsection{Thickness shear mode}

In case of a partially electroded $Y$-cut resonator with the electric field applied parallel to the $x_{2}$ axis, only the strain $S_{6}$ and stress $T_{6}$ do not vanish. The piezoelectric relation shown in Eq. (6) can be expressed as

$$
\begin{aligned}
& T_{6}=\hat{c}_{66} S_{6}+e_{26} E_{2}, \\
& D_{2}=e_{26} S_{6}+\hat{\varepsilon}_{22} E_{2} .
\end{aligned}
$$

Under the assumption of a harmonic time dependence of an electric field, Eq. (19), Newton's equation of motion may be transformed analogously to the case of length extensional mode of vibration. The resulting equation is found to be (Mason, 1964)

$$
\left(\hat{c}_{66}+\frac{e_{26}^{2}}{\hat{\varepsilon}_{22}}\right) \frac{\partial^{2} u_{1}}{\partial x_{2}^{2}}+\omega^{2} \rho u_{1}=0 .
$$

Here, a new property, the piezoelectrically stiffened shear modulus $\bar{c}$ is introduced

$$
\begin{aligned}
\bar{c} & =\hat{c}_{66}+\frac{e_{26}^{2}}{\hat{\varepsilon}_{22}}=c_{66}+\frac{e_{26}^{2}}{\varepsilon_{22}+\sigma^{2} / \varepsilon_{22} \omega^{2}} \\
& +j \omega\left(\eta+\frac{\sigma_{2}}{1+\sigma_{2}^{2} / e_{26}^{2} \omega^{2}}\right) .
\end{aligned}
$$

This shear modulus depends on the piezoelectric and dielectric coefficients, as well as on the electromechanical losses, i.e., electric conductivity and viscosity. The consequences of these relations are discussed elsewhere (Fritze, 2011b).

Using these equations, the electric impedance of the thickness shear resonator is obtained

$$
Z=\frac{1}{j \omega C_{0}}\left[1-\hat{k}_{t}^{2} \frac{2}{\hat{v}} \tan \left(\frac{\hat{v}}{2}\right)\right]
$$

with coupling factor $\hat{k}_{t}^{2}=e_{26}^{2} / \hat{c}_{66} \hat{\varepsilon}_{22}$ and the reduced wave velocity $\hat{v}=\omega a \sqrt{\rho / \hat{c}}$. Here, $a$ denotes the thickness of the resonator.

\subsubsection{Face shear mode}

The face shear vibration of a $Y$-cut resonator is determined by the elastic compliance $s_{44}$. This two-dimensional motion cannot be described by a physical model which has a straightforward analytical solution. Therefore, only an approximate solution for the resonance frequency $f_{\mathrm{r}}$ of such a resonator as presented in (Ikeda, 1990; Bechmann, 1951) is used:

$f_{\mathrm{r}}=\frac{\kappa \Theta}{\pi l} \sqrt{\frac{1}{\rho s_{44}}}$.

Here, $\kappa$ is a solution of transcendental equation $\tan \kappa+\kappa=0$, and $\Theta$ is a correction factor defined as

$$
\Theta=1-\frac{1}{2 \kappa}\left(\frac{\kappa^{2}-2}{\kappa^{2}+2}\right)^{3 / 2} \sqrt{\frac{s_{11}+s_{33}}{2 s_{44}}} .
$$

\subsection{Parameters of SAW propagation}

The parameters of SAW propagation, like velocity $v$, propagation loss $\alpha$ and coupling coefficient $k^{2}$, are calculated from the tensor data, obtained from the impedance measurements of BAW resonators as described in Sect. 2.3.

For this purpose the equations of motion

$$
\begin{aligned}
& \rho \frac{\partial^{2} u_{i}}{\partial t^{2}}=\frac{\partial T_{i j}}{\partial x_{j}}, \\
& \frac{\partial D_{i}}{\partial x_{i}}=0,
\end{aligned}
$$

with $i, j=1,2,3$, have to be solved together with the piezoelectric Eq. (6) for the semi-infinite substrate crystal of the given cut. For this, the Green function method described in (Peach, 1995, 2001; Koskela, 1998) is used. The poles and zeros of the Green function component $G_{44}$ on the complex slowness space $s$ correspond to surface acoustic waves, propagating on a free and on a metallized surface, respectively. A complex pole

$G_{44}^{p}=\frac{K}{s-s_{0}}$

where the value $s_{0}=s_{0}^{\prime}+j s_{0}^{\prime \prime}$ is associated with a SAW, which propagates on the free surface with the velocity $v_{0}=$ $1 / s_{0}^{\prime}$. The attenuation is

$\alpha=2 \pi f \cdot \frac{s^{\prime \prime}}{s^{\prime}} \cdot 20 \cdot \lg e[\mathrm{~dB} / \mu \mathrm{s}]$.

Here, $f$ and $e$ are the frequency of the SAW and the Euler number, respectively.

\subsection{Parameters of SAW propagation extracted from the SAW measurements}

Special test SAW devices are designed for the experimental determination of the surface acoustic wave parameters $v$, $\alpha$ and $k^{2}$. These are four delay lines of two different lengths. Two of them have free propagation paths, the other two propagation paths are metallized as shown in Fig. 2. 


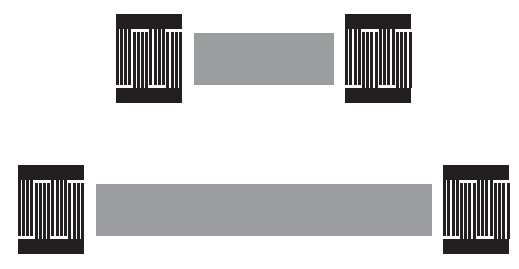

Figure 2. Delay lines of different length.

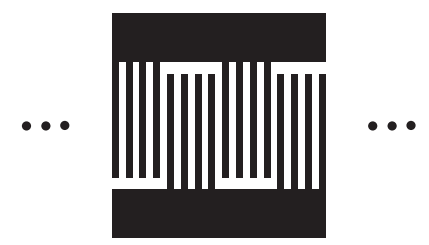

Figure 3. IDT with eight electrodes per period.

Thereby, identical input and output interdigital transducers (IDTs) with eight electrodes per electrical period are used (see Fig. 3).

They can be regarded as multi-electrode transducers with double spatial sampling. As shown in (Engan, 1975), the transfer function $S_{21}$ of such IDT has four pass bands (four harmonics) with central frequencies corresponding to $1 / 4 f_{0}$, $3 / 4 f_{0}, 5 / 4 f_{0}, 7 / 4 f_{0}$, where $f_{0}=v /(2 p)$. The pitch $p$ corresponds to the period of the electrode in the IDT. In our case the transfer function $S_{21}$ shows the 1st, 3rd, 5th and 7th harmonics at about 150, 450, 750 and $1050 \mathrm{MHz}$ (Fig. 4).

During signal processing the difference between time delay of long and short delay lines at each harmonic is obtained very precisely (Shrena et al., 2008). This allows the determination of the group velocity based on the known length difference between both delay lines. The influence of the IDTs is, therefore, automatically eliminated. The results for the group velocity of SAWs on the free surface and on the metallized surfaces are extrapolated to zero frequency. This way, the phase velocity for SAW on the free LGS surface $v_{0}$ and on the short-circuited LGS surface $v_{\mathrm{m}}$ (i.e., metallized surface without mass loading by the metal film) is determined. Further, the electromechanical coupling coefficient $k^{2}$ is obtained from the phase velocities $v_{0}$ and $v_{\mathrm{m}}$ using the Ingebrigtsen relation (Ingebrigtsen, 1972)

$k^{2}=-2 \cdot \frac{v_{\mathrm{m}}-v_{0}}{v_{0}}$.

The propagation losses $\alpha$ at the harmonic frequencies are calculated to be

$\alpha=\frac{1}{\Delta t} \cdot 20 \lg \frac{\left|S_{21}^{\text {long }}\right|}{\left|S_{21}^{\text {short }}\right|}[\mathrm{dB} / \mu \mathrm{s}]$,

where $S_{21}^{\text {long }}$ and $S_{21}^{\text {short }}$ are the transfer function of the long and short delay line at the harmonic frequency, respectively,

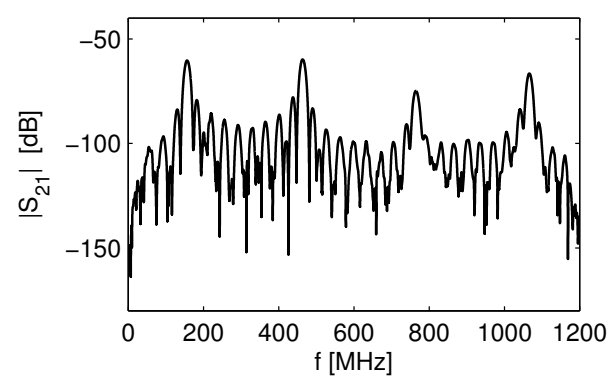

Figure 4. Transfer function $S_{21}$ of a delay line with four harmonics (after signal processing).

and $\Delta t$ is the difference of delay times of the two delay lines for this harmonic.

\section{Experimental}

\subsection{Sample preparation}

\subsubsection{Crystal cuts}

For the determination of the bulk properties of langasite crystals several orientations as shown in Fig. 1 are used. Two rectangular $10 \times 10 \times 0.5 \mathrm{~mm}^{3} X$ - and $Z$-cut plates are used for impedance measurements at low frequencies. The $Y$-cut sample has the same dimensions and operates in the face shear mode of vibration. A circular $Y$-cut sample of $0.27 \mathrm{~mm}$ in thickness is excited in the thickness shear mode. For determination of most components of the elastic compliance tensor, four differently rotated $10 \times 2 \times 0.5 \mathrm{~mm}^{3}$ rods with electrodes perpendicular to the $X$ axis are prepared. During the machining of langasite rods, the angle $\varphi$ could not be adjusted as precise as other dimensions of the sample with typical uncertainty of about $\pm 1^{\circ}$. In order to overcome this limitation, the samples are characterized at room temperature and the elastic compliance tensor is calculated. The comparison of the resulting tensor with literature data and previous pulse-echo measurements on the same crystals enable precise determination of the cut angles.

For the SAW measurements two cuts with Euler angles $(0$, $\left.138.5,26.6^{\circ}\right)$ and $\left(0,30.1,26.6^{\circ}\right)$ are selected. They show large $k^{2}$ and zero power flow angle (PFA) (Naumenko and Solie, 2001; da Cunha and Fagundes, 1999; Plessky et al., 1998). In addition, the first cut is temperature compensated around room temperature, which makes it attractive for many applications. Because of that, this cut is commercially available from many distributors.

\subsubsection{Electrodes}

\section{BAW samples}

All samples are coated with platinum electrodes by pulsed laser deposition (PLD). The thickness of this electrodes is 
about $250 \mathrm{~nm}$. A titanium adhesion layer of a few nanometers is applied.

\section{SAW devices}

The metallization of the SAW test devices is done by SAW Components Dresden GmbH using the lift-off technique. Thin Pt layers of 45 and $75 \mathrm{~nm}$ are deposited. A $\mathrm{Zr}$ film of $4 \mathrm{~nm}$ in thickness is taken as adhesion layer. Dewetting of the thin Pt film above that temperature determines a temperature limit for the measurements. In contrast to the BAW samples, thicker Pt layers cannot be used due to increasing reflection and scattering effects at the IDTs which distort the signal and cause additional losses.

\subsection{Setup and data evaluation}

\section{BAW measurements}

The electric impedance at low frequencies is measured using a Solartron 1260 gain-phase analyzer to determine the electrical conductivity. The impedance in the vicinity of the resonance frequency of all BAW resonators is acquired using a HP 5100A network analyzer. For high-temperature measurements in artificial air containing $20 \% \mathrm{O}_{2} / \mathrm{Ar}$, a gas-tight furnace with temperature varying between 20 and $900^{\circ} \mathrm{C}$ at the rate of $2 \mathrm{~K} \mathrm{~min}^{-1}$ is used. In case of the conductivity determination, the temperature is held constant before each measurement in order to equilibrate the samples.

The measured impedance data are further fitted using the physical models of the corresponding resonators in order to obtain the components of stiffness and elastic compliance tensors. It is obvious that the number of free parameters for the fit procedure must be much smaller than the total number of parameters, i.e., 16 for the one-dimensional model of thickness shear resonators. Reasonable fits can be, in general, expected if the number of free parameters does not exceed three or four (Fritze, 2011b). As a starting point at room temperature, variables such as the sample dimensions, density and electrode areas are determined precisely. Thermal expansion of the material, which influences the dimensions and density, is taken into account. Further, the thickness of electrodes is measured precisely using the surface profiler. In order to calculate the mass load, their density is assumed to be $100 \%$ of the theoretical Pt density as they are prepared by PLD.

The determination of remaining parameters is performed in several steps:

1. Impedance measurements of $X$ - and $Z$-cut plates at low frequencies provide information about the dielectric coefficient and the conductivity in the form of complex coefficients $\hat{\varepsilon}_{11}$ and $\hat{\varepsilon}_{33}$.

2. The fit of analytically solved piezoelectric equations uses the dielectric coefficient and conductivity from step (1).
The determination of elastic properties of langasite involves several crystal orientations and modes of vibration. First, the four differently oriented rods shown in Fig. 1 provide the coefficients $s_{11}(T), s_{14}(T), s_{33}(T)$ and $\left(s_{44}(T)+s_{13}(T)\right)$. Second, the rectangular $Y$-cut plate vibrating in face shear mode provides the elastic compliance $s_{44}(T)$, and thus, enables the separation of $s_{13}(T)$ compliance from length-extensional measurements. Finally, the round $Y$-cut resonator excited in the thickness-shear mode of vibration provides the stiffness coefficient $c_{66}(T)$ used to calculate the elastic compliance $s_{12}(T)$.

The data evaluation applied here potentially delivers unreliable absolute values due to, e.g., residual stray capacitance of the high-temperature sample holder. To overcome this problems and to verify the models, fit results are compared with preliminary pulse-echo measurements performed on the same langasite single crystal.

\section{SAW measurements}

Two delay lines - one long and one short - are positioned on one chip. They are connected to the same ports and are measured simultaneously. The chip is mounted on a holder and is connected to the RF-flange of the high-temperature furnace via two rigid coaxial cables made of steel tubes with ceramic husked steel wires. The other side of the RF-flange is connected by standard RF coaxial cables to the network analyzer, which measures the transfer function $S_{21}$. Before starting measurements, the atmosphere inside the furnace is changed to nitrogen in order to reduce oxidation effects. The sample is heated at a slow continuous ramp of $50 \mathrm{~K} \mathrm{~h}^{-1}$ up to the final temperature of $800^{\circ} \mathrm{C}$. The transfer function $S_{21}$ is measured in temperature steps of $5 \mathrm{~K}$.

A specially developed signal processing algorithm utilizing cross-correlations, is used for the determination of the precise time delay and for extracting SAW propagation parameters as described in detail in (Shrena et al., 2008, 2009). As shown in (Shrena et al., 2009), using a cross-correlation algorithm gives better accuracy for the determination of time delays in comparison to other methods.

\section{Results and discussion}

\subsection{Materials parameters}

\subsubsection{Electric properties at elevated temperatures}

The conductivity of the $X$ - and $Z$-cut langasite plates is summarized in Fig. 5. The anisotropy of the conductivity is clearly visible.

\subsubsection{Full set of piezoelectric and elastic properties}

As a result of the procedure described in Sect. 3.2, the complete elastic compliance tensor and the piezoelectric tensor as a function of temperature, $s(T)$ and $d(T)$, are available. 


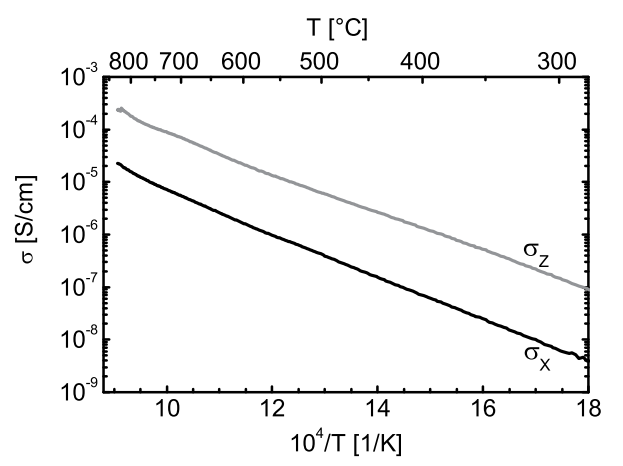

Figure 5. Conductivity of LGS as function of temperature.

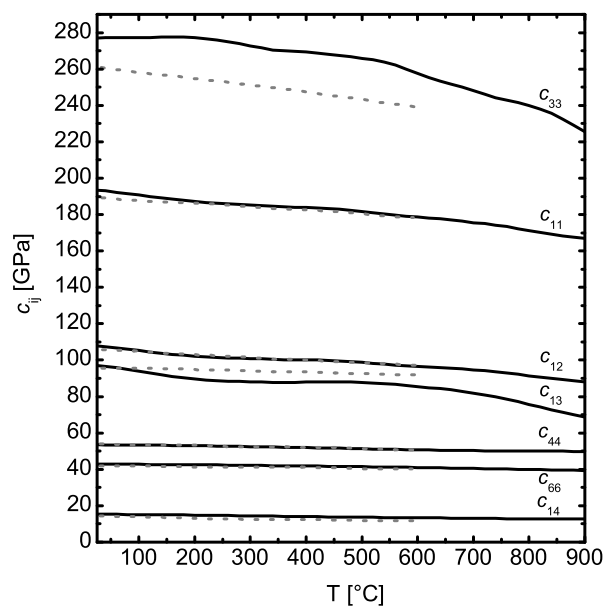

Figure 6. The full set of elastic stiffness coefficients of langasite as function of temperature calculated from BAW measurements (solid line) in comparison with the data obtained by Weihnacht et al. (2012) (dotted line).

They are used to calculate the stiffness tensor $\boldsymbol{c}(T)$ and the piezoelectric tensor $\boldsymbol{e}(T)$ using the respective relations. The elastic stiffness and the piezoelectric coefficient as function of temperature are summarized in Figs. 6 and 7, respectively.

A comparison of elastic data from this work with data obtained by Weihnacht et al. (2012). Weihnacht et al. (2012) show a very good agreement except for the stiffness coefficient $c_{33}$ which differs by about $5 \%$ from the referenced work. In case of the purely acoustic method as used by (Weihnacht et al., 2012) it is possible to measure the phase velocity along the $x_{3}$ axis directly. However, in case of resonant measurement, the excitation of resonant vibration in this direction is not possible. As shown in Eq. (7), all components of piezoelectric tensor $\boldsymbol{d}$ or $\boldsymbol{e}$ related to the $x_{3}$ direction equal zero. Therefore, the stiffness coefficient $c_{33}$ as well as the elastic compliance $s_{33}$ can be determined using the effective stiffness or compliance resulting from a vibration of a rotated crystal. In the related Eq. (10), the term $\sin ^{4} \varphi$, dominates only for $45^{\circ}$ rotated rod and nearly vanishes for all other rotation angles. For this reason the coefficients $s_{33}$ and $c_{33}$ are

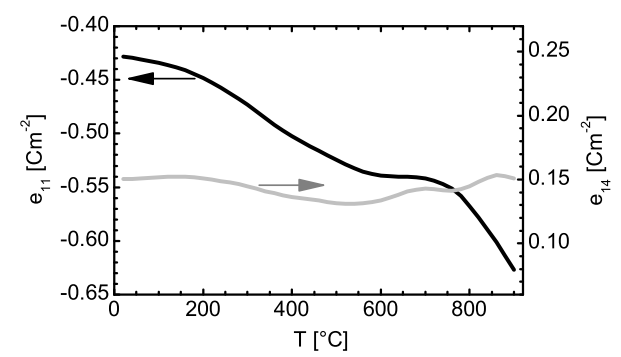

Figure 7. The piezoelectric coefficient of langasite as function of temperature.

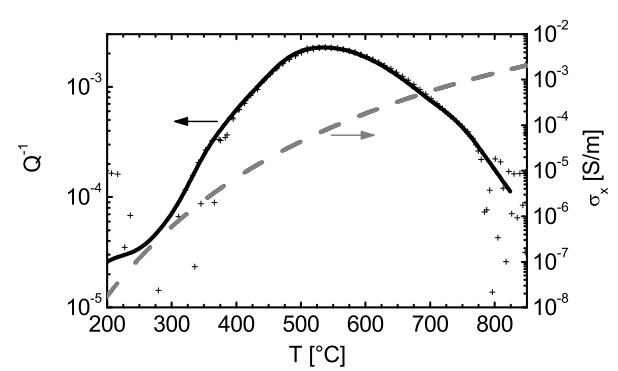

Figure 8. The inverse resonant quality factor $Q^{-1}$ and the electric conductivity $\sigma_{X}$ of langasite rod operated at $220 \mathrm{kHz}$ in length extensional mode of vibration.

most error-prone which explains the small discrepancy mentioned above.

\subsubsection{Temperature-dependent loss}

As already mentioned in Sect. 2.1, the electrical conductivity and mechanical properties such as viscosity contribute to the losses in high-temperature piezoelectric materials. The viscosity of the langasite rod with the angle $\varphi=0$ (see Fig. 1) expressed in form of the inverse resonant quality factor $Q^{-1}$ (see Eq. 2) is calculated from the physical fit. These data and the electric conductivity $\sigma_{X}$ are shown in Fig. 8. Here, a maximum of loss around $500{ }^{\circ} \mathrm{C}$ is clearly visible.

\subsection{SAW properties}

The characterization of SAW devices is carried out up to about $730^{\circ} \mathrm{C}$ where the $\mathrm{Zr} / \mathrm{Pt}$-metallization failed through de-wetting and decomposition into droplets as visualized in Fig. 9.

The phase velocities $v_{0}$ and $v_{\mathrm{m}}$ on free and metallized surfaces for the cut $\left(0,138.5,26.6^{\circ}\right)$ are shown in Fig. 10. The obtained phase velocities at room temperature and coefficients of frequency for a second order polynomial fit are given together with literature data in Table 1.

Figure 11 shows the electromechanical coupling coefficient calculated from Eq. (28) for the propagation geometry with Euler angles $\left(0,138.5,26.6^{\circ}\right)$ and from the tensor data of BAW resonators. The difference between BAW and 


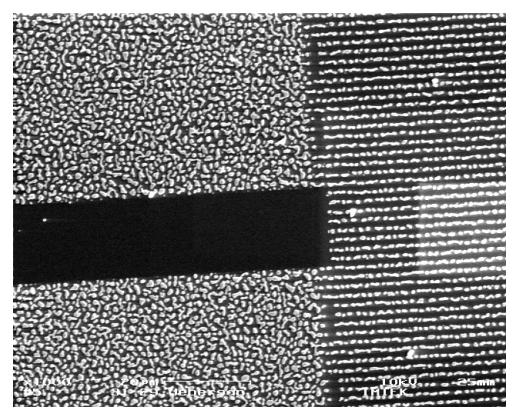

Figure 9. Dewetted metal layer after heating up to $800^{\circ} \mathrm{C}$.

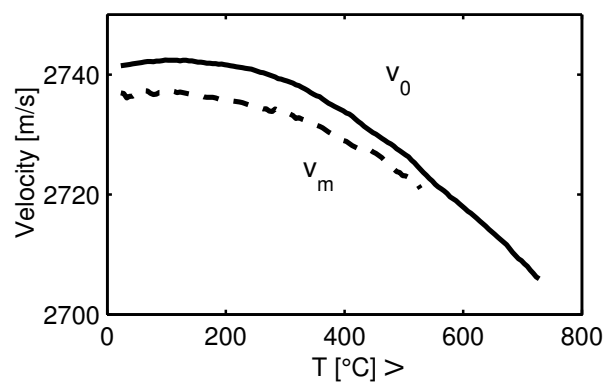

Figure 10. Phase velocity of Rayleigh waves on free surface $v_{0}$ (solid line) and on metallized surface $v_{\mathrm{m}}$ (dotted line), cut $(0,138.5$, $\left.26.6^{\circ}\right)$.

SAW data for $k^{2}$ are caused by the uncertainty of the SAW method. As shown in Eq. (28), $k^{2}$ results from subtraction of two large numbers which were obtained by an extrapolation procedure. Furthermore, room temperature data are in satisfactory agreement with the corresponding electromechanical coupling coefficient, $k^{2}=0.33$, calculated from the tensor data given in (Kosinski et al., 2001), and with data obtained by other authors, $k^{2}=0.5$ (Fachberger et al., 2004) and $k^{2}=0.34$ (Naumenko and Solie, 2001).

The propagation losses $\alpha$ on free surfaces for both investigated cuts of langasite are shown in Fig. 12. Similar to the langasite rods operated in length-extensional mode of vibration, SAW devices exhibit a local propagation loss maximum at around $520^{\circ} \mathrm{C}$ for all measured frequencies.

The propagation loss on the metallized surface shown in Fig. 13 increases monotonically. However, they exhibit sharp changes of the slope approximately at the same temperature as for the free surface.

\subsection{Stability of the electrodes for BAW and SAW resonators}

LGS-based SAW and BAW devices exhibit an operation temperature limit caused by the stability of the platinum electrodes. It is found, that their lifetime drops drastically as their thickness decreases. This effect is described by, e.g., Firebaugh et al. (1998). Due to this effect the SAW devices used in this work failed at about $730^{\circ} \mathrm{C}$, whereas the BAW res-
Table 1. Comparison of our measurement results with literature data. In case of (Kosinski et al., 2001), the values are calculated using tensor data.

\begin{tabular}{lrrr}
\hline cut & $v_{0}, \mathrm{~m} \mathrm{~s}^{-1}$ & $\mathrm{TCF} 1, \mathrm{ppm} \mathrm{K}^{-1}$ & $\mathrm{TCF} 2, \mathrm{ppb} \mathrm{K}^{-2}$ \\
\hline$\left(0,138.5,26.6^{\circ}\right)$ & 2741.9 & 1.5 & -41 \\
$\left(0,30.1,26.6^{\circ}\right)$ & 2464.6 & 28 & -39 \\
\hline & \multicolumn{4}{c}{ results of other authors } \\
\hline & $2741.8^{\mathrm{a}}$ & $-7^{\mathrm{a}}$ & $-51^{\mathrm{a}}$ \\
& $2734^{\mathrm{b}}$ & $1^{\mathrm{b}}$ & - \\
& $2743^{\mathrm{c}}$ & - & - \\
\hline$\left(0,138.5,26.6^{\circ}\right)$ & $2462.1^{\mathrm{a}}$ & $31^{\mathrm{a}}$ & $-69^{\mathrm{a}}$ \\
& $2392.1^{\mathrm{c}}$ & - & - \\
\hline
\end{tabular}

${ }^{\text {a }}$ Fachberger et al. (2004), ${ }^{\mathrm{b}}$ Naumenko and Solie (2001), ${ }^{\mathrm{c}}$ Kosinski et al. (2001).

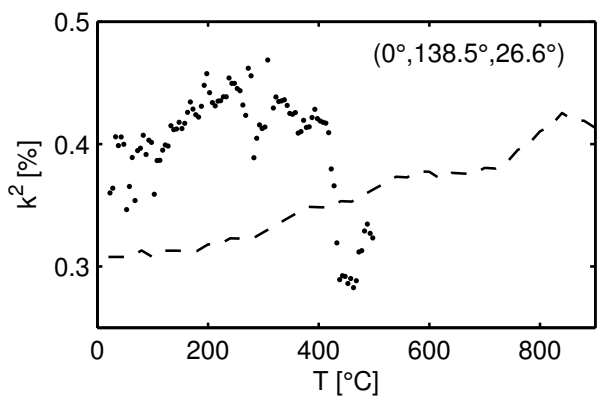

Figure 11. Coupling coefficient $k^{2}$ for Rayleigh waves on cut $(0$, $\left.138.5,26.6^{\circ}\right)$, calculated with tensor data from BAW measurements (dashed lines) and extracted from SAW measurements (points).

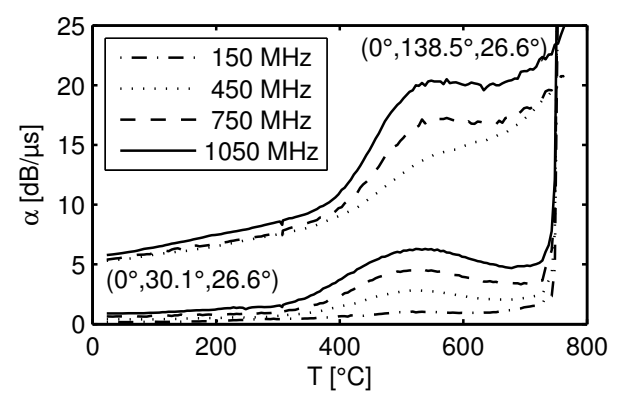

Figure 12. The propagation loss in $[\mathrm{dB} / \mu \mathrm{s}]$ of Rayleigh waves on free surface on both cuts $\left(0,138.5,26.6^{\circ}\right)$ and $\left(0,30.1,26.6^{\circ}\right)$, measured at different frequencies.

onators wearing thicker electrodes could be operated up to $900^{\circ} \mathrm{C}$. If more stable electrodes, obtained by, e.g., screen printing, are used, even higher operation temperatures for BAW devices are demonstrated (Fritze, 2011b).

Above the mentioned temperatures, de-wetting of the metal layer is observed, as shown in Fig. 9 for SAW structures. Several attempts have been made to investigate and possibly reduce this effect. Da Cunha et al. conducted a series of experiments with different layer combinations for the metallization to extend the temperature limits for the SAW devices (da Cunha et al., 2007; Behanan et al., 2015). Most 


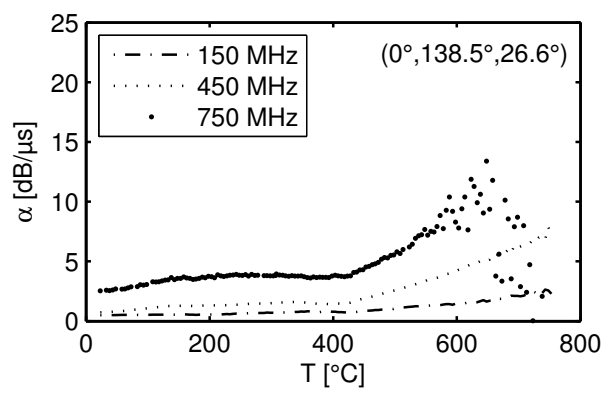

Figure 13. The propagation loss in $[\mathrm{dB} / \mu \mathrm{s}]$ of Rayleigh waves on metallized surface on cut $\left(0,138.5,26.6^{\circ}\right)$ for different frequencies.

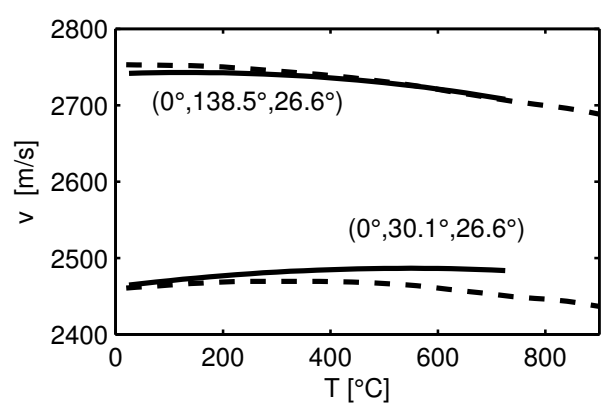

Figure 14. Comparison of Rayleigh wave velocity on free surface $v_{0}$ obtained from SAW and BAW measurements (solid and dashed lines, respectively).

recent attempts to minimize the degradation of electrodes and prolong the lifetime of SAW elements include use of metal alloys as well as high-temperature ceramic electrodes. Richter et al. report about successful operation of SAW elements at $800^{\circ} \mathrm{C}$ for several hours using $\mathrm{Ti} / \mathrm{Pt}$ coated langasite (Richter et al., 2011). Further attempts include application of thin protective layers, like $\mathrm{Al}_{2} \mathrm{O}_{3}$. An example of successful application of multi-layer stack metallization consisting of $\mathrm{Al} / \mathrm{Al}_{x} \mathrm{O}_{y} / \mathrm{Pt}$ for the high-temperature sensors is given in (Wall et al., 2015).

\subsection{Correlation of SAW-measured phase velocities with calculated BAW data}

In order to compare the bulk and surface acoustic wave properties, the full set of temperature-dependent materials data is determined using BAW resonators and applied to calculate the phase velocity $v_{0}$ of SAW devices with Euler angles of $\left(0,138.5,26.6^{\circ}\right)$ and $\left(0,138.5,26.6^{\circ}\right)$. As seen in Fig. 14 , the results of the calculation and the measured data are in good agreement especially for the $\left(0,138.5,26.6^{\circ}\right)$ cut, which confirms the validity of the fitted parameters and the calculated materials data.

\section{Conclusions}

A method of materials data extraction from measured data of BAW resonators is developed for piezoelectric crystals. All components of the stiffness, elastic compliance and piezoelectric tensors for langasite are determined in the temperature range from 20 to $900^{\circ} \mathrm{C}$. Furthermore, the conductivity of the crystal in $X$ and $Y$ directions and the quality factor $Q$ for resonant deformations in the $X$ direction are obtained as function of temperature. For these experiments, BAW resonators with frequencies in the range 200 $5000 \mathrm{kHz}$ are used. The obtained elastic stiffness tensor is in good agreement with data published by other authors, except for the stiffness coefficient $c_{33}$, which exhibits a deviation of about $5 \%$ from the literature data.

Based on the obtained tensor data, the phase velocity and the coupling coefficient for SAW propagation of the crystal cuts $\left(0,138.5,26.6^{\circ}\right)$ and $\left(0,30.1,26.6^{\circ}\right)$ is calculated. The same parameters are extracted from measurements of SAW delay lines up to $730^{\circ} \mathrm{C}$, which are fabricated using the crystal cuts mentioned above and operated at frequencies from 150 up to $1050 \mathrm{MHz}$.

Values for the velocity of SAW propagation and coupling coefficients obtained from SAW and from BAW measurements show a good agreement. SAW devices exhibit a local maximum of propagation loss at around $520^{\circ} \mathrm{C}$. BAW rods exhibit a maximum of losses at the same temperature.

Thin film Pt electrodes for BAW and SAW devices limit the maximum temperature of measurement to 900 and $730^{\circ} \mathrm{C}$, respectively. The operating temperature may be significantly increased by application of protective layers and thick electrodes. The latter is, however, feasible in the case of BAW resonators only.

Acknowledgements. The authors thank the German research foundation (Deutsche Forschungsgemeinschaft, DFG) for financial support and the Energy Research Center Niedersachsen.

Edited by: J. Zosel

Reviewed by: two anonymous referees

\section{References}

Bardong, J., Schulz, M., Schmitt, M., Shrena, I., Eisele, D., Mayer, E., Reindl, L., and Fritze, H.: Precise measurements of BAW and SAW properties of Langasite in the temperature range from $25 \mathrm{C}$ to $1000 \mathrm{C}$, in: 2008 IEEE International Frequency Control Symposium, 326-331, 2008.

Bechmann, R.: Contour Modes of Square Plates excited Piezoelectrically and Determination of Elastic and Piezoelectric Coefficients, P. Phys. Soc. Lond., B64, 323-337, 1951.

Behanan, R., Moulzolf, S., Call, M., Bernhardt, G., Frankel, D., Lad, R., and da Cunha, M.: Thin films and techniques for SAW sensor operation above $1000^{\circ} \mathrm{C}$, in: 2013 Joint UFFC, EFTF and PFM Symposium, 1013-1016, 2015. 
Bungo, A., Jian, C., Yamaguchi, K., Sawada, Y., Uda, S., and Pisarevsky, Y.: Analysis of Surface Acoustic Wave Properties of Rotated Y-Cut Langasite Substrate, Jpn. J. Appl. Phys., 38, 32393243, 1999.

da Cunha, M. P. and Fagundes, S. D. A.: Investigation on recent quartz-like materials for SAW applications, IEEE Trans. on Ultrasonics, IEEE Int. Ferro., 46, 1583-1590, 1999.

da Cunha, M. P., Moonlight, T., Lad, R., Bernhard, G., and Frankel, D. J.: Enabling very high temperature acoustic wave devices for sensor \& frequency control applications, in: 2007 IEEE Ultrasonics Symposium, 2107-2110, 2007.

Engan, H.: Surface acsoustic wave multilevel transducers, IEEE T. Son. Ultrason., 6, 395-401, 1975.

Fachberger, R., Holzheu, T., Riha, E., Born, E., Pongratz, P., and Cerva, H.: Langasite and langatate nonuniform material properties correlatedto the performance of SAW devices, in: Frequency Control Symposium and PDA Exhibition, 2001, Proceedings of the 2001 IEEE International, 235-239, 2001.

Fachberger, R., Bruckner, G., Hauser, R., Ruppel, C., Biniasch, J., and Reindl, L.: Properties of radio frequency Rayleigh waves on langasite at elevated temperatures, in: Proc. 2004 IEEE Ultrasonics Symposium, 1223-1226, 2004.

Firebaugh, S. L., Jensen, K. F., and Schmidt, M. A.: Investigation of High-Temperature Degradation of Platinum Thin Films with an In Situ Resistance Measurement Apparatus, J. Microelectromech. S., 7, 128-135, 1998.

Fritze, H.: High temperature piezoelectric materials: Defect chemistry and electro-mechanical properties, J. Electroceram., 17, 625-630, 2006.

Fritze, H.: High-temperature piezoelectric crystals and devices, J. Electroceram., 26, 122-161, 2011a.

Fritze, H.: High-temperature bulk acoustic wave sensors, Meas. Sci. Technol., 22, 012002, 2011b.

Fritze, H. and Tuller, H. L.: Langasite for High Temperature Bulk Acoustic Wave Applications, Appl. Phys. Lett., 78, 976-977, 2001.

Fritze, H., Schulz, M., Seh, H., and Tuller, H.: Sensor applicationrelated defect chemistry and electromechanical properties of langasite, Solid State Ionics, 177, 2313-2316, 2006.

Fukuda, T., Takeda, P., Shimamura, K., Kawanaka, H., Kumatoriya, M., Murakami, S., Sato, J., and Sato, M.: Growth of new langasite single crystals for piezoelectric applications, in: Applications of Ferroelectrics, 1998. ISAF 98, Proceedings of the Eleventh IEEE International Symposium on, 315-319, 1998.

Ikeda, T.: Fundamentals of piezoelectricity, Oxford University Press, Oxford, UK, 1990.

Ingebrigtsen, K. A.: Analysis of interdigital transducers, IEEE T. Son. Ultrason., 403-407, 1972.

Kosinski, J. A., Pastore Jr., R. A., Bigler, E., Pereira da Cunha, M., Malocha, D. C., and Detaint, J.: A Review of Langasite Material Constants from BAW and SAW Data: Toward and Improved Data Set, in: IEEE International Frequency Control Symposium, 278286, 2001.

Koskela, J.: Modeling SAW devices including mass-loading effects, Helsinki University of Technology, Helsinki, Finland, 1998.

Malocha, D., da Cunha, M., Adler, E., Smythe, R. C., Frederick, S., Chou, M., Helmbold, R., and Zhou, Y. S.: Recent Measurements of Material Constants versus Temperature for Langatate, Langanite and Langasite, in: Frequency Control Symposium and Ex- hibition, 2000. Proceedings of the 2000 IEEE/EIA International, 200-205, 2000.

Mason, W. P.: Physical Acoustics, Principles and Methods, vol. 1A, Academic Press, New York, USA, 1964.

Meitzler, A., Tiersten, H., Warner, A., Berlincourt, D., Couqin, G., and Welsh III, F.: IEEE Standard on Piezoelectricity, ANSI/IEEE Std, IEEE, 1988.

Naumenko, N. and Solie, L.: Optimal cuts of langasite, $\mathrm{La}_{3} \mathrm{Ga}_{5} \mathrm{Sio}_{14}$ fpr SAW Devices, IEEE T. Ultrason. Ferr., 38, 530-537, 2001.

Peach, R.: A general Green function analysis for SAW devices, in: Proc. IEEE Ultrason. Symp., 221-225, 1995.

Peach, R.: On the existence of surface acoustic waves on piezoelectric substrate, IEEE T. Ultrason. Ferr., 49, 1308-1320, 2001.

Plessky, V., Koskela, J., Lehtonen, S., and Salomaa, M.: Surface transverse waves on langasite, in: 1998 IEEE Ultrasonics Symposium Proceedings, 139-142, 1998.

Richter, D., Sakharov, S., Forsén, E., Mayer, E., Reindl, L., and Fritze, H.: Thin Film Electrodes for High Temperature Surface Acoustic Wave Devices, Procedia Engineering, 25, 168-171, 2011.

Schneider, T., Richter, D., Doerner, S., Fritze, H., and Hauptmann, P.: Novel impedance interface for resonant high-temperature gas sensors, Sensor. Actuat. B-Chem., 111, 187-192, 2005.

Schulz, M. and Fritze, H.: Electromechanical properties of langasite resonators at elevated temperatures, Renew. Energ., 33, 336-341, 2008.

Sherrit, S. and Mukherjee, B. K.: The Use of Complex Material Constants to Model the Dynamic Response of Piezoelectric Materials, in: IEEE Ultrasonics Symposium, 633-640, 1998.

Shrena, I., Eisele, D., Mayer, E., Reindl, L., Bardong, J., and Schmitt, M.: SAW-relevant material properties of langasite in the temperature range from 25 to $750{ }^{\circ} \mathrm{C}$ : New experimental results, in: IEEE Ultrasonics Symposium, 209-212, 2008.

Shrena, I., Eisele, D., Bardong, J., and Reindl, L.: High precision signal processing algorithm to evaluate the SAW properties as a function of temperature, in: IEEE Ultrasonics Symposium, 863866, 2009.

Smits, J. G.: Influence of Moving Domain Walls and Jumping Lattice Defects on Complex Material Coefficients of Piezoelectrics, IEEE T. Son. Ultrason., SU-23, 168-174, 1976.

Thiele, J. A. and Pereira da Cunha, M.: High temperature LGS SAW gas sensor, Sensor. Actuat. B-Chem., 113, 816-822, 2006.

Tuller, H. and Fritze, H.: High-temperature balance, US Patent $6.370 .955,2002$.

Wall, B., Gruenwald, R., Klein, M., and Bruckner, G.: A $600^{\circ} \mathrm{C}$ Wireless and Passive Temperature Sensor Based on Langasite SAW-Resonators, in: AMA Conferences 2015 - SENSOR 2015 and IRS2 2015, 19-21 May 2015, Nuremberg, Germany, 390395, doi:10.5162/sensor2015/C3.3, 2015.

Weihnacht, M., Sotnikov, A., Schmidt, H., Wall, B., and Grünwald, R.: Langasite: High Temperature Properties and SAW Simulations, in: 2012 IEEE International Ultrasonics Symposium Proceedings, 1549-1552, doi:10.1109/ULTSYM.2012.0387, 2012. 